\title{
Anabases
}

ANABASES Traditions et réceptions de l'Antiquité

19 | 2014

Varia

\section{Madagascar avant Madagascar : l'île Ménouthias des anciens et les premières représentations de l'île de Saint Laurent}

\section{Bianca Maria Altomare}

\section{OpenEdition}

\section{Journals}

Édition électronique

URL : http://journals.openedition.org/anabases/4680

DOI : $10.4000 /$ anabases.4680

ISSN : 2256-9421

Éditeur

E.R.A.S.M.E.

\section{Édition imprimée}

Date de publication : 1 avril 2014

Pagination : $227-241$

ISSN : $1774-4296$

\section{Référence électronique}

Bianca Maria Altomare, "Madagascar avant Madagascar : I'île Ménouthias des anciens et les premières représentations de l'île de Saint Laurent », Anabases [En ligne], 19 | 2014, mis en ligne le 01 avril 2017, consulté le 20 octobre 2019. URL : http://journals.openedition.org/anabases/4680 ; DOI : $10.4000 /$ anabases.4680 


\section{Madagascar avant Madagascar : l'île Ménouthias des anciens et les premières représentations de l'île de Saint Laurent ${ }^{1}$}

Il fut un temps où Ortygie voguait sur les ondes; à présent elle est fixe. La nef Argo craignit les Symplégades, jouets des vagues, dont les assauts venaient se briser sur elles; à présent ces îles demeurent immobiles et résistent aux vents.

Ovide, Métamorphoses XV, 336-339

Comme les îles nommées par Ovide, Madagascar a toujours vogué dans l'imaginaire géographique de l'océan Indien au fil du temps. Iconographiquement, cette île a aussi vogué sur les cartes représentant l'océan Indien, avant de se " fixer » avec une forme et une situation précises et réelles sur la carte de Cantino, datée de 1502, quelques années après la découverte de l'île, faite par Diogo Dias le jour de la Saint Laurent $1500^{2}$.

1 Le présent travail trouve sa source dans une intervention au Séminaire "Océan Indien " du CEMAF, le 23 février 2012. Je voudrais remercier D. Marcotte (directeur de recherche d'élection), J.-Y. Sarrazin, E. Vagnon et E. Vallet, ansi que tous ceux qui m'ont aidée dans ce parcours d'installation et d'intégration en France. Cet article leur est dédié.

2 J. Fontvieille, "À propos de la découverte de Madagascar ", Bulletin de l'Académie Malgache 39-41 (1963) p. 30-31 ; H. Le Louvier Aumont de Bazouges, Madagascar, lîle de Nulle-Part ailleurs, L'Harmattan, Paris, 1999. Pour un récit de la découverte de Madagascar, voir les Decadas Da Asia de João de Barros/Diego Do Couto (Dec. VII, liv. IV, cap. V, p. 312-314). 
En effet, avant cette date, plusieurs auteurs occidentaux, de l'Antiquité au Moyen Âge, ont parlé d'une grande île au large des côtes africaines, en lui donnant plusieurs noms : Phébol, Ménouthias, Diab, Madagascar ${ }^{3}$. Parallèlement, les cartes géographiques antérieures à 1500 (exempli gratia, le Globe de Martin Behaim de 1492) ont représenté cette terre, encore inconnue aux marins occidentaux ${ }^{4}$, en lui attribuant une forme imaginaire et un nom, Madagascar, qui restera le nom officiel du " petit continent ». Le présent travail portera sur la " double mythologie », littéraire et cartographique, relative à Madagascar. On analysera tout d'abord les témoignages littéraires concernant l'île, de l'Antiquité jusqu'à la mention dans le Livre des Merveilles de Marco Polo. On regardera ensuite de plus près ses représentations cartographiques. Il sera d'abord question des représentations imaginaires de l'île, liées au récit de Marco Polo : le planisphère de Fra' Mauro (1459) et le globe de M. Behaim (1492) ; on étudiera ensuite l'apparition de la "vraie " île de Madagascar/Saint Laurent sur les cartes du XvI ${ }^{\mathrm{e}}$ siècle, de Cantino (première image de Madagascar après sa découverte par les marins portugais) à Mercator. On pourra ainsi mieux saisir le processus de construction d'une imago mundi entre $\mathrm{XV}^{\mathrm{e}}$ et $\mathrm{XVI}^{\mathrm{e}}$ siècle. En effet, à cette époque si fertile en découvertes, les savants durent se confronter avec les représentations antiques de l'œkoumène dans le but de les renouveler ${ }^{5}$ : "Cartographie marine et cartographie ptoléméenne - enrichie à la lumière aussi bien des découvertes littéraires que des connaissances issues des voyages au-delà de l'œkoumène antique - fournissent le socle graphique et géographique sur lesquels les cartographes construisent l'imago mundi'."

3 Ce qui reste très intéressant, c'est le fait que l'île était appelée différemment par les navigateurs arabes et par ses habitants. Cependant, elle ne fut baptisée que par les navigateurs qui l'abordèrent et la naissance du nom Madeigascar, reste une énigme (voir infra).

4 L'île était bien connue des marins arabes, et la carte d'El-Idrisi (1153) est le plus ancien document connu et conservé la représentant. Voir G. FerRAND, Relations de voyages et textes géographiques arabes, persans et turcs relatifs à l'Extrême Orient du VIII au XVII' siècle, Leroux, Paris, 1913 ; G. Ferrand, P. Vérin, " Madagascar ", Encyclopédie de l'Islam V (1986), p. 943-948.

5 P. Gautier Dalché, La géographie de Ptolémée en Occident, IVe-XVI siècle, Brepols, Turnhout, 2009, p. 235-236; M. Milanesi, Tolomeo sostituito, Studi di storia delle conoscenze geografiche nel XVI secolo, Milano, Unicopii, 1984.

6 Humanisme et découvertes géographiques, dossier coordonné par N. Bouloux, P. Gautier Dalché et A. Cattaneo, Vincennes, Presses universitaires de Vincennes, 2010, p. 48. 


\section{Témoignages littéraires de l'Antiquité : Phébol et Ménouthias}

Dans les témoignages les plus anciens, il est souvent question d'une île, Ménouthias, vulgo identifiée avec Madagascar ${ }^{7}$. On parle aussi de Phébol (Pseudo-Aristote, De mundo : il s'agit d'un hapax) ou des îles du Soleil (dans la description attribuée à Iamboulos, rapportée par Diodore de Sicile). Il est impossible de pencher pour une identification de cette île avec Madagascar ; cependant, relire et regrouper les textes nous permettra de mieux saisir le développement d'une imago de cette partie de l'océan Indien.

\section{Ps.-Aristote, De mundo 393 b 15}

Dans un article paru en 1975 dans la revue Omaly sy Anio ${ }^{8}$, Yves Janvier aborde la question d'une éventuelle connaissance de Madagascar par les marins grecs et romains. Dans son énumération des auteurs qui auraient parlé de Madagascar, il cite le traité De Mundo (393b15), attribué à Aristote, sans analyser le texte grec. Il convient ici d'en donner une traduction :

En partant de l'Est, l'Océan entre aussi dans les terres, et forme d'un côté la mer Indienne, le golfe Persique, et la mer Erythrée. De l'autre côté, en partant du même point d'orient, il baigne la Caspie et l'Hyrcanie, et occupe une vaste étendue au nord de la PalusMéotides. Ensuite, resserrant peu à peu la terre habitée, au-dessous de la Scythie et de la Celtique, il revient vers les Gaules, et de là aux colonnes d'Hercule, au-delà desquelles est l'Océan. Dans cette mer se trouvent les deux grandes îles Britanniques, Albion et Hierna, plus grandes que celles que nous avons nommées ci-dessus : elles sont situées immédiatement au-dessus des Celtes.

Il y en a au-delà de l'Inde, qui ne sont pas moins considérables : Taprobane, qui a sa position oblique au continent; celle de Phébol, qui est vers le golfe Arabique.

7 À ce propos, les ouvrages fondamentaux, quoique dépassés, restent A. Grandidier, Histoire de la géographie de Madagascar, Imprimerie Nationale, Paris, 1885 et G. Gravier, La Cartographie de Madagascar, A. Challamel, Paris, 1896. Voir aussi les différents hypothèses faites par A. BERTHELOT, L'Afrique saharienne et soudanaise - Ce qu'en ont connu les Anciens, Payot, Paris, 1927, p. 218, 229, 289-290, 393-394 ; E. STесHOW, "Kannte das Altertums die Insel Madagascar ? ", in Petermanns geographische Mittelungen, Justus Perthes, Gotha, 1844, p. 84 ; H. Deschamps, Histoire de Madagascar, Paris, BergerLevrault, $1972^{4}$, p. 30-34.

8 Y. JANVIER, "La géographie gréco-romaine a-t-elle connu Madagascar ? Le point de la question », Omaly sy Anio 1-2 (1975), p. 11-41. 
Selon Janvier, qui n'a cependant pas lu le texte grec, il s'agit d'une île nommée par souci de symétrie et inexistante dans l'horizon géographique grec. Reale ${ }^{9}$ reconnaît qu'elle n'est pas identifiable ${ }^{10}$ et postule une interpolation postérieure. La mention des deux grandes îles se trouve en effet isolée dans le contexte et la description géographique subit une rupture du fil logique qui guide le discours. Il faut ajouter en outre que le nom "Phébol » est un hapax et une évidente corruption de toponyme, comme l'indique la crux des éditeurs pour ce passage qui reste très controversé ${ }^{11}$.

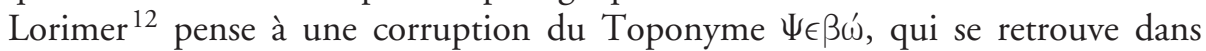
Strabon XVII, 2, 3 et qui est identifié avec le lac Tana en Abyssinie. Étienne de Byzance parle aussi d'une $\Psi \in \beta \omega \prime$, " contrée lointaine d'Éthiopie " (il s'agirait d'une citation d'Aristagoras et d'Artémidore) ${ }^{13}$.

\section{Le marin Iamboulos (Diodore de Sicile, Bibliothèque historique II, 55-56)}

Janvier consacre une analyse beaucoup plus détaillée au témoignage de Diodore de Sicile II, 55-56 (seconde moitié du I ${ }^{\text {er }}$ siècle de notre ère $\left.{ }^{14}\right)$. Il s'agit du récit d'Iambulos, marin autrement inconnu, capturé par des brigands arabes, peut-être au $\mathrm{III}^{\mathrm{e}}$ siècle av. J.-C. ${ }^{15}$. Après quatre mois de navigations, il serait arrivé sur l'Île du Soleil pour y séjourner sept ans, bien accueilli par les indigènes.

D’une part, il paraît clair que le récit présente des caractéristiques qui le rapprochent du genre de l'utopie et de la paradoxographie ${ }^{16}$ (exempli gratia, le fait que les

9 Il trattato "Sul cosmo per Alessandro " attribuito ad Aristotele: monografia introduttiva, testo greco con traduzione a fronte commentario, bibliografia ragionata e indici, a cura di G. Reale, A. P. Bos, Vita e pensiero, Milano, 1995, p. 277.

10 Pour une identification Phébol/Madagascar, voir W. CAPElle, Der Schrift von der Welt, ein Weltbild im Umriss aus dem 1. Jahrhundert nach Chr., E. Diederichs, Jena, 1907, p. 539 ; A.-J. Festugière, La Révélation d'Hermès Trismégiste, vol. 2 : Le Dieu cosmique, J. Gabalda, Paris, 1949, 465 ; J. TRICOT, Traité du ciel, suivi du Traité pseudo-aristotélicien Du monde, Paris, J. Vrin, 1948, p. 185.

11 Les manuscrits transmettent $\Phi \circ \beta \in \alpha, \quad \Phi \in \rho \beta \omega, \quad B \in \phi o \lambda, \quad \Phi \in \beta \circ \lambda o_{0}, \quad \Phi \in \beta o \nu, \quad \Phi \in \lambda \beta \circ \lambda$. Voir W.L. Lorimer, Some Notes on the Text of Pseudo-Aristotle "De Mundo", Oxford, Oxford University Press, 1925, p. 37.

12 LORIMER, Some notes, p. 37.

13 Voir aussi J. Hudson, Geographiae Veteris Scriptores Graeci Minores Cum Interpretatione Latina, Dissertationibus, ac Annotationibus, IV, Sheldon, Orford, 1717, p. 38 ; C. SAUMAISE, Claudii Salmasii Plinianae exercitationes in Caji Julii Solini Polyhistora. Item Caji Julii Solini Polyhistor ex veteribus libris emendatus, Paris, Morelli, 1629, p. 111.

14 Diodore de Sicile, Bibliothèque Historique, tome II, livre II, texte établi et traduit par B. EcK, Paris, Les Belles Lettres, 2003 ; voir surtout les p. XL-XLVI.

15 W. Kroll, "Iambulos ", Pauly Wissowa, IX. 1, col. 681-683.

16 Grandider, Berthelot et Deschamps n'ont pas pris en compte l'île comme une éventuelle mention de Madagascar ; diamétralement opposée la position de Stechow. Voir aussi : 
habitants connaissent un automne perpétuel, ni trop chaud ni trop froid ; qu'ils ont les os flexibles et la langue fendue, et une vie longue et exempte de maladies, se terminant par un suicide volontaire) ; de l'autre, il ne faut pas négliger des informations qui paraissent assez réalistes ${ }^{17}$, notamment la situation de l'île par rapport aux côtes africaines, la durée du voyage calculée sur la base du renversement de la mousson, l'absence d'ombre à midi et les jours constamment égaux aux nuits (climat équatorial).

Toute identification paraît bien sûr hasardeuse et le récit dans son intégralité nous porte à conclure que l'île avait certainement été atteinte, du moins sporadiquement, mais elle n'avait jamais été explorée systématiquement.

\section{Ptolémée, Marcien d'Héraclée et Étienne de Byzance : Ménouthias}

C'est toutefois l'île Ménouthias qui a été prise en considération pour une éventuelle identification avec Madagascar. Elle est mentionnée par l'auteur du Périple de la Mer Érythrée ( $\mathrm{I}^{\mathrm{er}}$ siècle de notre ère), Ptolémée (II ${ }^{\mathrm{e}}$ siècle), Marcien d'Héraclée ( $\mathrm{V}^{\mathrm{e}}$ siècle) et Étienne de Byzance ( $\mathrm{VI}^{\mathrm{e}}$ siècle).

De nombreuses hypothèses d'identification ont été formulées : Zanzibar, Pemba, Madagascar. Probablement, le toponyme mentionné dans le Périple de la Mer Érythrée $^{18}$ ne correspond pas à celui dont les autres géographes parlent. En effet, les

E. De Flacourt, C. Allibert, Histoire de la grande Isle Madagascar, Paris, Karthala, 2007, p. 460.

17 Pour une identification avec Madagascar, voir aussi F.F. SCHWARZ, "The itinerary of Iambulus-utopianism and history", in Indology and Law, Studies in Honour of J. Duncan and M. Derret, Steiner Franz Verlag, Wiesbaden, 1993, p. 43 ; M. WinIarCZYK, "Das Werk des Jamboulos. Forshungsgeschichte (1550-1988). Ein Interpretationsversuch », Rheinisches Museum 140, 2 (1997), p. 139-141.

18 Voir The Periplus Maris Erythraei, text with introd., transl., and comment., by L. CASSON, N.J. Princeton University Press, Princeton, 1989; The Periplus of the Erythraean Sea : with some Extracts from Agatharkhidès "On the Erythraean Sea ", transl. [from Greek] and ed. by G.W.B. Huntingford, London, Hakluyt Society, 1980 ; M. Horton, «The Periplus and East Africa ", Azania 25 (1990), p. 95-99. L'île Ménouthias mentionnée par Ptolémée est à 335 milles du Cap Prasos. En réalité, la distance entre Madagascar et le continent africain est de 454 milles. Selon le Périple, Ménouthias serait beaucoup plus proche des côtes africaines. Voir aussi J.C. HéBert, Madagascar et Malagasy, Histoire d'un double nom de baptème, Bulletin de Madagascar, secrétariat d'État à l'information et au Tourisme, Paris, 1971, n. 302-303 ; J. DesAnges, Recherches sur l'activité des Méditerranéens aux confins de l'Afrique, VIe siècle avant J.-C.-IV après J.-C., Atelier national reprod. Lille, th. univ. Lille 3, 1982 ; J. Reade, The Indian Ocean in Antiquity, Kegan Paul International, London-New York, 1996 . R. MAunY, "Le périple de la mer Erythrée et le problème du commerce romain en Afrique au sud du Limes ", Journal de la Société des Africanistes 38 (1968), p. 19-34, en particulier p. 27, écarte complètement une identification avec Madagascar : "L'île de Menuthias, citée également par Ptolémée, serait identifiable de préférence à Pemba ( $v^{\mathrm{e}}$ siècle) à $350-400 \mathrm{~km}$ de Patta-Lamu, distance qui peut 
données divergent irrémédiablement : latitude, longitude, distance des côtes africaines. Parmi toutes ces hypothèses, il est impossible d'identifier Ménouthias avec Madagascar et d'affirmer que les géographes grecs et romains connurent l'île.

Dans les témoignages que nous venons de présenter, il s'agit clairement de passages corrompus, de mentions assez vagues concernant les distances qui séparent l'île des côtes ou encore d'informations imprécises sur la direction de navigation à prendre. D'ailleurs, il faut remarquer que les dimensions de l'île ne sont pas données. Il pourrait dès lors s'agir d'une des nombreuses îles qui ponctuaient l'océan Indien. Un détail supplémentaire est donné par Marcien qui la situe aux limites du monde connu.

Voici les textes concernés :

a) Périple de la Mer Érythrée $15^{19}$

Au-delà d'Opone, la côte se dirige toujours vers le sud. Il y a d'abord les deux Apokopa, le petit et le grand Apokopa ; cette côte est dépourvue de ports mais il y a des endroits où les navires peuvent ancrer, la côte étant escarpée; ce trajet est de six jours en direction sud-ouest. Puis viennent la petite et la grande Aigialos pendant encore six jours et après cela, dans l'ordre indiqué, les dromoi d'Azania dont la première s'appelle Sarapion, la suivante Nikon et ensuite plusieurs estuaires et autres ancrages, l'un après l'autre, demandant chaque fois un arrêt et un trajet d'un jour, sept en tout, jusqu'aux îles Pyralaoi et ce qui est appelé Diôruchos (le chenal), au-delà duquel, un peu plus au sud-sud-ouest, après deux courses d'un jour et d'une nuit le long de la côte ausanatique, est l'âle de Ménouthias ${ }^{20}$ à environ trois-cent stades de la terre ferme, basse et boisée, où se trouvent des rivières et de nombreuses espèces d'oiseaux et la tortue de montagne.

b) Ptolémée, Géographie, IV, 8, 2

Le Cap Prasos ${ }^{21}$ se trouve à $80^{\circ} 15^{\circ} \mathrm{S}$

Près de celui-ci, en direction Nord-Est, se trouve l'âle Ménouthias,

Dont les coordonnées sont : $85^{\circ} 12^{\circ} 30^{\prime} S^{22}$

effectivement être couverte en 2 jours $1 / 2$ de navigation. Zanzibar ( $\mathrm{vI}^{\mathrm{e}}$ siècle) est déjà à $500 \mathrm{~km}$; quant à Mafia (Monfiyen), elle semble à écarter car les $650 \mathrm{~km}$ qui la séparent de Patta-Lamu seraient difficiles à couvrir pendant le même laps de temps ». Enfin, selon C. AlliberT, "Le mot "Komr" dans l'océan Indien (avec une note sur Qanbalu) ", Études Océan Indien 31 (2001), p. 13-33, l'île Ménouthias mentionnée par le Périple et par Marcien, correspondrait au même toponyme.

19 GGM I, 267-270.

20 Le manuscrit transmet $\epsilon\llcorner\mathrm{\epsilon} \in \nu \eta \delta\llcorner\omega \mu \mu \in \nu 0 v \theta \in \sigma\llcorner a \zeta$ (cf. GGM I, 270).

21 Le Cap Delgado.

22 A. Stuckelberger, Ptolemaios Handbuch der Geographie, Griechisch-Deutsch, Hrsg. von Alfred Stückelberger und Gerd Grasshoff, Basel, I, Schwabe AG, 2006, p. 459, identifie l'île avec Pemba ou Zanzibar. 
c) Marcien d'Héraclée, Périple de la Mer Extérieure, I, $13^{23}$

Après la mer Rouge, en changeant la route vers le Sud et tout en tenant encore la terre à droite, on rencontre ensuite le mont Elefas et la région des Aromes ; après celle-ci vient la région Barbare et la mer nommée Barbare, dans laquelle il y a plusieurs golfes, et les « passages de Azania ». Le plus célèbre entre les golfes qui s’y trouvent est celui nommé Apokopa, et encore à la suite le Petit Littoral et le Grand Littoral, auxquels suit un autre grand golfe jusqu'au promontoire Rhapton. Après ces lieux se trouve celle qu'on nomme Mer Brève et le golfe qui l'entoure ; elle est habitée par les barbares Anthropophages. À la fin du golfe se dresse le très grand promontoire qu'on appelle Cap Prasos et, pas loin de celui-ci, l'île Ménuthias.

d) Étienne de Byzance, s.v. Ménouthis ${ }^{24}$

Ille d'Éthiopie

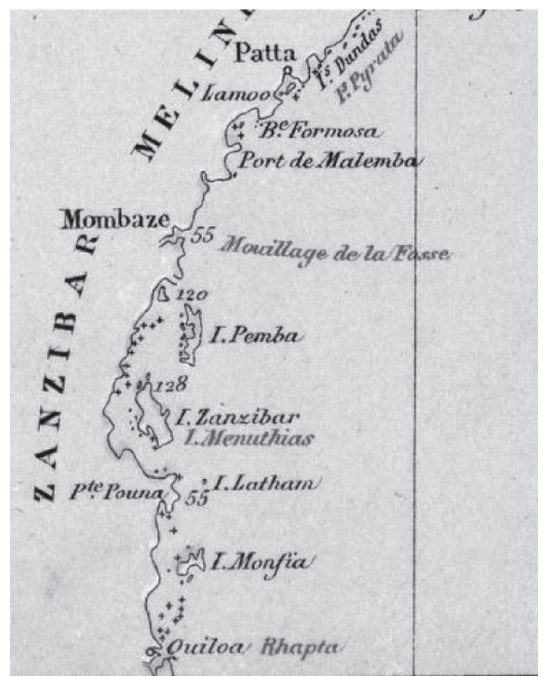

a.

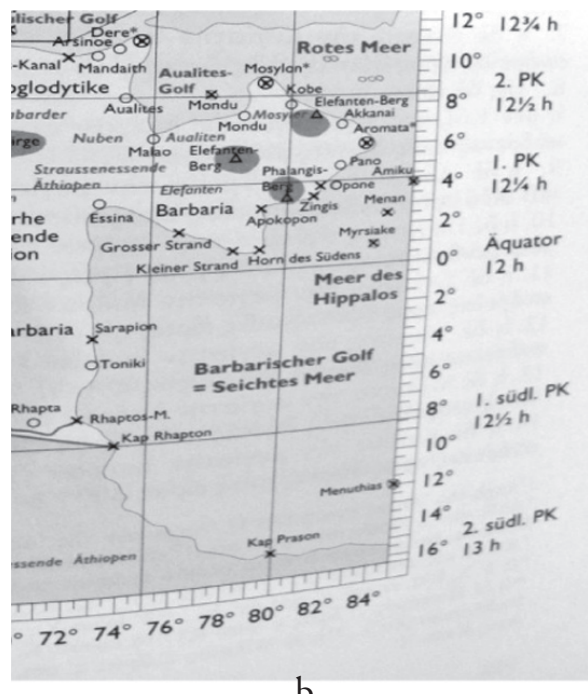

b.

Fig. 1 Divergences dans la localisation et l'identification de Ménuthias:

a. Carte générale de la mer des Indes, pour servir à l'intelligence du périple de la mer Erythrée dressée par M. Calixte Alary, sous la direction de M. le vice-amiral Jurien de La Gravière,

Paris, imprimerie de Dufrénoy, 1884 ;

b. Stuckelberger, Ptolemaios Handbuch, I, p. 459.

$23 G G M$ I, 563.

24 Stephani Byzantii Ethnicorum quae supersunt, ex recensione Augusti Meinekii, Berlin, G. Reimer, 1849. 


\section{Témoignages littéraires au Moyen Âge : le Livre des Merveilles de Marco Polo}

Véritable summa des connaissances géographiques au Moyen Âge, le Livre des Merveilles, récit du voyage que Marco Polo avait effectué entre 1274 et 1295, de Venise jusqu'en Chine, contient une mention de la grande île de Madaigascar. Il s'agit d'un témoignage extrêmement important : bien que l'île mentionnée ne soit pas Madagascar (les éditeurs concordent pour une identification avec Mogadiscio), on doit surtout à Marco Polo (et aux cartographes qui s'inspirent de lui, Behaim et Fra'Mauro in primis), le baptême de ce toponyme ${ }^{25}$. Dans le chapitre 35, présenté de suite, il raconte :

Après avoir quitté l'île de Scoira et naviguant du côté du midi pendant mille milles, on vient à Madeigascar, qui est mise au nombre des plus riches îles du monde. On dit qu'elle contient quatre mille milles de tour ; les habitants sont mahométans. Ils n'ont point de roi, mais ils sont gouvernés par quatre des plus anciens. Cette île produit beaucoup plus d'éléphants qu'aucun pays du monde. Il y a une île nommée Zanzibar qui fait un grand trafic d'ivoire, car en tout le monde je ne pense pas qu'il y ait une si grande quantité d'éléphants que dans ces deux îles. On ne mange point dans cette île d'autre viande que celle de chameau, laquelle chair est fort saine aux habitants ; il y a une multitude presque infinie de ces animaux dans cette île. Il y a outre cela dans cette île des forêts de sandales et de bon rouge, dont on fait plusieurs ouvrages. On prend aussi dans la mer de grandes baleines, d'où l'on tire de l'ambre. Il y a des lions, des léopards, des cerfs, des daims, des chevreuils et plusieurs autres sortes d'animaux et d'oiseaux propres à la chasse. Enfin on $\mathrm{y}$ trouve diverses espèces d'oiseaux dont on n'a jamais entendu parler chez nous. Plusieurs marchands viennent en cette île à la faveur du flux de la mer. Car on peut venir en vingt jours de la province de Maabar à cette île de Madeigascar avec le flux de la mer; mais on a de la peine à en sortir; et il faut quelquefois trois mois pour surmonter les difficultés de ce flux, parce que la mer porte toujours vers le midi avec beaucoup d'impétuosité.

Les manuscrits transmettent le nom Madeigascar ${ }^{26}$, unanimement identifié avec Mogadiscio ${ }^{27}$. Cette île encore imaginaire sera néanmoins à la base d'un certain nombre de représentations cartographiques. À partir du XVe siècle, l'île de Madagascar dont Marco Polo nous parle apparaitra dans les cartes ou dans les globes en face de la corne d'Afrique. Elle aura une forme irréelle, qui ne correspond pas à ses véritables contours, encore inconnus du monde occidental. Un des exemples les plus frappants

25 Voir Allibert, " Le mot Qomr ", p. 14 ; Hébert, Madagascar et Malagasy, p. 583-613; G. Ferrand, "Trois étymologies arabico-malgaches ", Mémoires de la Société de linguistique de Paris 13 (1905), p. 413-430.

26 Et nombreuses variations : Madeigasca, Magdeigascar, Madagastar, Mandeschar, Mandeigaster, Magastar. Voir Grandidier, Histoire de la géographie, p. 24.

27 Le manuscrit $\mathrm{F}$ présente Madeigascar, $\mathrm{Z}$ présente Mogdasio. 
est constitué par le globe de Martin Behaim, daté de 1492. Il montre la forme de l'œkoumène à la veille des découvertes de Christophe Colomb et s'inspire abondamment du Devisement du monde de Marco Polo. Le cartographe de Nuremberg place l'île au large, en direction Sud-Est, près de Zanzibar. Les légendes qu'on peut y lire sont tirées du Livre des Merveilles.

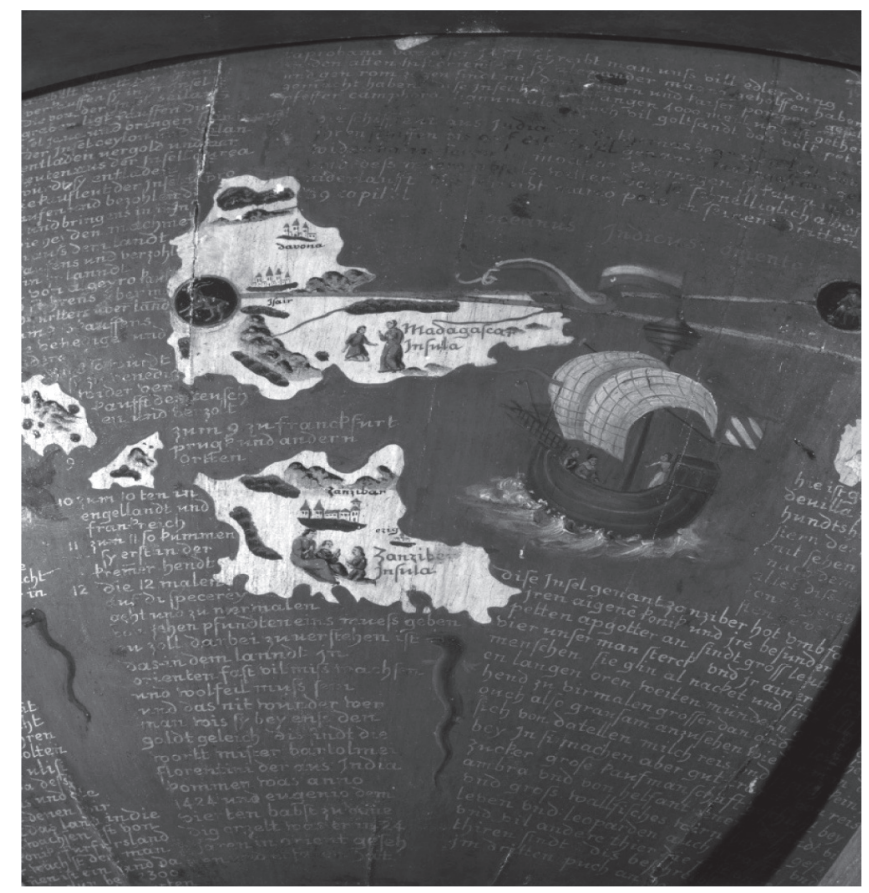

Fig. 2 L'île de Madagascar dans le globe de Marin Behaim, Nuremberg, 1492 (copyright BnF, Cartes et Plans).

Quelques années plus tard, les découvertes portugaises vont donner une morphologie réelle à ce fantasme littéraire. C'est alors que l'imaginaire et le réel se rencontrent : le nom qui avait résonné dans les pages des livres et dans les cartes du XV siècle correspond enfin à une terra cognita! Giovanni Battista Ramusio fut le premier à identifier la grande île récemment découverte avec celle décrite par Marco Polo. Dans son ouvrage monumental, Navigazioni e viaggi ${ }^{28}$, on peut lire : "Della grand'isola di Magastar, ora detta di San Lorenzo. "Selon Pelliot ${ }^{29}$, « l'application d'un nom erroné remonte

28 Troisième volume. Cf. M. MilanesI, Giovanni Battista Ramusio, Navigazioni e viaggi, volume terzo, Einaudi, Torino, 1980, p. 286.

29 A.C. Moule, P. Pelliot, Marco Polo, The description of the World, George Routhledgs \& Sons, London, 1938, p. 595. 
finalement à l'époque de Ramusio. Mais le fait demeure : une faute de copiste dans les manuscrits de Polo a donné pour jamais son nom à la grande île ».

\section{Représentations cartographiques : l'humanisme et la nouvelle imago mundi}

\section{Fra' Mauro}

Le Devisement du monde de Marco Polo fut à la base d'un autre monumentum de la cartographie du Xve siècle : la mappemonde de Fra' Mauro, datée de 1459. Dans cette énorme mappemonde circulaire, on peut voir l'état des connaissances au milieu $\mathrm{du} \mathrm{XV}^{\mathrm{e}}$ siècle. Le Sud est en haut de la carte, l'océan Indien est représenté comme une mer ouverte. Les sources du cartographe sont les géographes grecs anciens mais surtout Marco Polo et les navigateurs arabes, comme on l'apprend des nombreuses et précieuses légendes qui enrichissent la carte :

Certains pourraient croire étrange que je parle de cette partie méridionale (scil. de l'œcoumène), presque entièrement inconnue aux anciens. Mais je leur réponds que tout ce dessin, du Nord au Sud, il m'est venu des gens qui y son nés, très religieux, et qui m’ont dessiné toutes ces provinces, les villes, les fleuves et les montagnes avec leurs noms.

Si l'on s'approche donc de la zone qui nous intéresse, on voit l'Afrique et, à côté d'elle, une île énorme, nommée Diab. Giovanni Battista Ramusio, dans son introduction au Livre des Merveilles de Marco Polo déjà citée, fait allusion à la carte de Fra' Mauro : "Si vede appresso l'isola di Magastar, ora detta di San Lorenzo, e quella di Zinzibar, delle quali messer Marco Polo parla ne' capitolii 35 e 36 des terzo libro, e molte altre particularità nelli nomi dell'isole orientali, che dapoi per Portughesi a' tempi nostri sono state scoperte ${ }^{30}$. "Pour lui, Madagascar et Diab sont une seule et même chose.

Les savants se sont longuement interrogés sur l'identité de Diab. Selon Zurla ${ }^{31}$, par exemple, il ne s'agirait pas d'une île mais d'un toponyme né des informations que Fra' Mauro avait obtenues des marins musulmans. En effet, à l'intérieur de l'île, il est question de toponymes tels que Soffala (Sofala, Mozambique) ou Chelue (Kilwi, Tanzanie) ; il s'agirait donc d'une représentation des régions extrêmes du sud ouest de l'île. Ce qui a fait pencher les éditeurs, notamment P. Falchetta ${ }^{32}$, pour une identifica-

30 P. FAlChETTA, Fra Mauro's world map, with a commentary and translations of the inscriptions, Brepols, Turnhout, 2006, p. 98.

31 P. Zurla, Il Mappamondo di fra Mauro camaldoles descritt e illustrato da D. Placido Zurla dello stess'Ordine, Venezia, 1806.

32 P. Falchetta, Fra Mauro's world map, p. 99. 
tion Diab $33 /$ Madagascar est la mention d'un canal qui sépare Diab du reste de l'Afrique (détail qu'on retrouve aussi dans Marco Polo et dans Ibn Majid ${ }^{34}$, en référence au Canal de Mozambique) ${ }^{35}$.

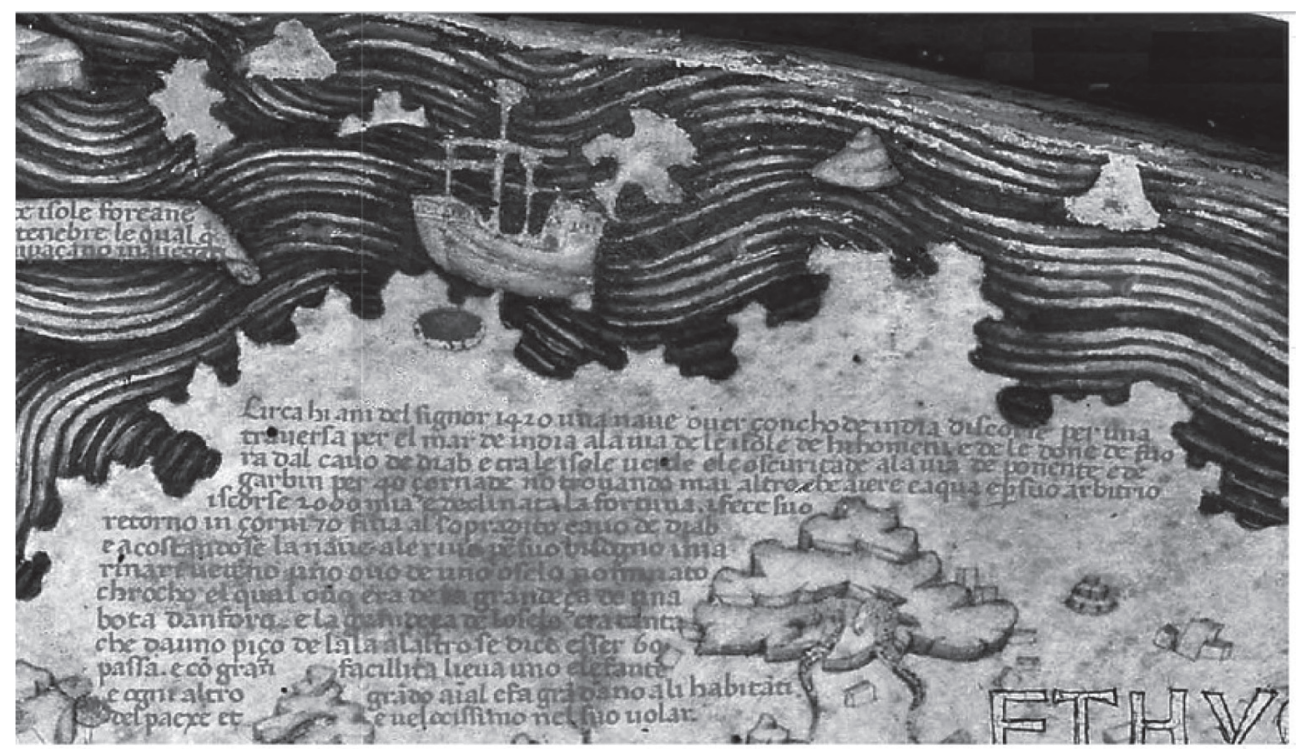

Fig. 3 : Les légendes qui figurent sur la représentation du Sud du continent africain dans la mappemonde de Fra' Mauro.

33 Pour l'étymologie, voir : Falchetta, Fra' Mauro's Worldmap, p. 99-100.

34 G. R. TiвbetTs, Arab Navigation in the Indian Ocean Before the coming of the Portuguese being a translation of Kitab al-Fawa'id fi usul al bahr wa'l-qawaid of Ahmand b. Majid al Najdi together with an introduction on the history of Arab navigation, notes on the navigational techniques and on the topography of the Indian Ocean, and a glossary of navigational terms, Royal Asiatic Society of Great Britain and Ireland, London, 1971, p. 208 et 218-219.

35 On peut lire à la note 49 : « Nota che questo cavo de diab è separato da abassia per uno canal, el qual è circundado da uno ladi e da l'altro de monti altissimi e albori si grandi e spesi che i fano quel canal oscuro, el qual ne la sua insidiafa uno çirolo (Agullhas) pericoloso per modo che se nave se ne abatesse la pericolerai. "Cf. aussi la note 19: "Circa hi ani del Signor 1420 una naue ouer çoncho de india discorse per una trauersa per el mar de india a la uia de le isole de hi homeni e de le done de fuora dal cauo de diab e tra le isole uerde e le oscuritade a la uia de ponente e de garbin per 40 çornade, non trouando mai altro che aiere e aqua, e per suo arbitrio iscorse 2000 mia e declinata la fortuna i fece suo retorno in çorni 70 fina al sopradito cauo de diab. E acostandose la naue a le riue per suo bisogno, i marinari ubi uno ouo de uno oselo nominato chrocho, el qual ouo era de la grandeça de una bota d'anfora, e la grandeça de l'oselo era tanta che da uno piço de l'ala a l'altro se dice esser 60 passa, e con gran facillità lieua uno elefante e ogni altro grando animal e fa gran dano a li habitanti del paexe et è uelocissimo nel suo uolar. " 


\section{Ptolémée renouvelé : Francesco Rosselli et Martin Waldseemuller}

Les représentations de Behaim et Fra' Mauro furent réalisées à l'aube des grandes découvertes. Durant cette période, l'imago mundi fut amplement renouvelée. Inévitablement, Ptolémée fut soumis à une véritable diorthosis au fur et à mesure que les contours de l'œecoumène s'élargissaient. C'est ainsi que, vers la deuxième moitié du $\mathrm{XV}^{\mathrm{e}}$ siècle, Ménouthias disparut des cartes ptoléméennes et devint Madagascar : il s'agissait encore, toutefois, d'une terra incognita, de l'île imaginaire dont il était question dans le Livre des merveilles.

L'exemple le plus frappant se trouve dans le planisphère de Francesco Rosselli $\left(1493^{36}\right)$. Cette carte aurait dû accompagner une édition de Ptolémée. Elle nous montre les contours de l'Afrique sur la base des découvertes de Bartolomeu Dias en 1487. Madagascar, encore inconnue, y occupe cependant une place importante. La morphologie est irréelle : il s'agit encore de l'héritage de la description de Marco Polo.

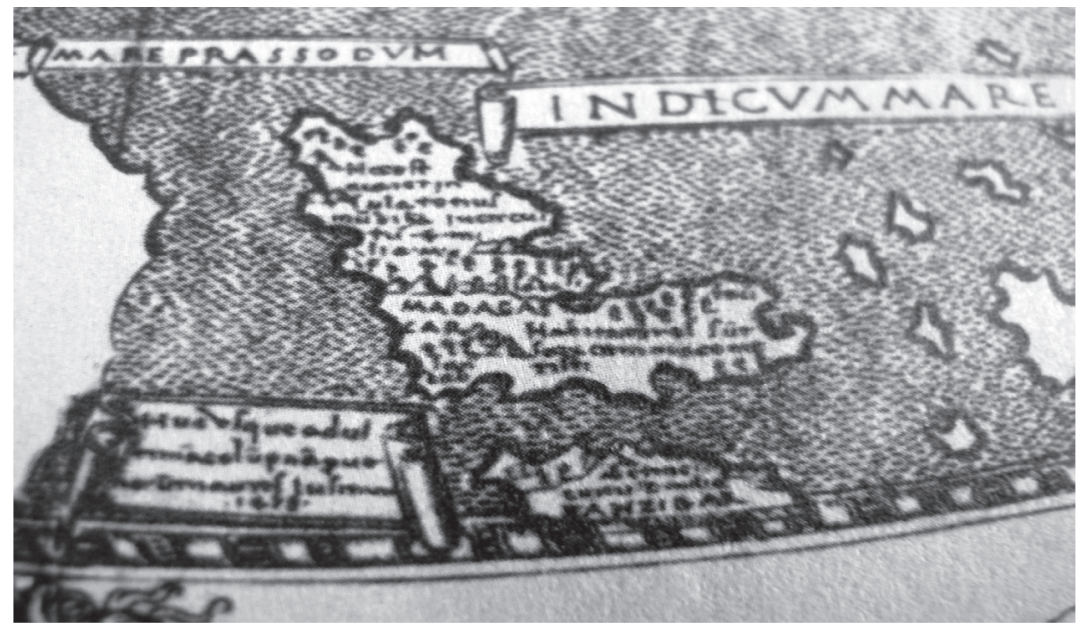

Fig. 4 : une île irréelle au large des côtes africaines :

Madagascar dans le Planisphère de Francesco Rosselli.

Un véritable changement apparaît dans les tabulae novae ajoutées à l'édition de Ptolémée de Strasbourg (1513), dessinées par Martin Waldseemuller (qui s’inspirait

36 Voir R.W. Shirley, The Mapping of the World, Early Printed World Maps 1472-1770, A. Saifer, New Holland, 1985, p. 17. Cette carte reprend le planisphère de Martelllus, 1490, qui cependant ne représente pas Madagascar. Cf. SHIRleY, The Mapping, p. XXIV. Voir aussi : R. Almagià, "On the cartographic works of Francesco Rosselli ", Imago mundi 8 (1951), p. 27-34, P. Gautier Dalché, La géographie de Ptolémée, p. 128. 
directement de son prédécesseur, Rosselli). Madagascar se donne à voir telle qu'elle avait été aperçue au large des côtes africaines :

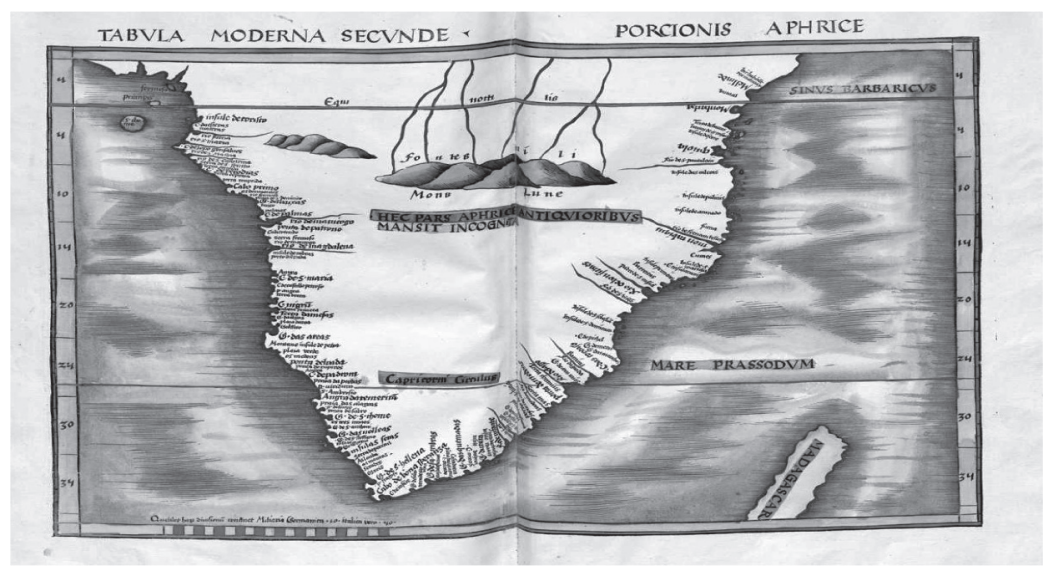

Fig. 5 : Madagascar dans la Tabula moderna secundae porcionis Africae de l'édition de Ptolémée (Strasbourg 1513).

\section{Le baptême cartographique de Madagascar : Cantino, Caverio, Mercator}

La découverte de Madagascar, survenue le jour de la Saint Laurent 1500, fut à l'origine de nombreuses cartes. Désormais, là où les cartographes $\mathrm{du} \mathrm{XV} \mathrm{V}^{\mathrm{e}}$ siècle plaçaient la Madagascar de Marco Polo et où les cartographes de Ptolémée situaient Ménouthias, on voit apparaître l'île Sao Lourenczo. Dans la carte de Cantino ${ }^{37}$, padron real réalisé en 1501, au lendemain des découvertes, l'île figure avec ses contours réels. Son nom est Comorbimam (transcription approximative du nom indien Comor-Diva, Île Comor), bien qu'elle soit située trop au Sud du continent africain. De cette carte dépend directement le Planisphère Nautique de Nicolo de Caverio (1506). Madagascar s'est enfin fixée sur les cartes. Au nom Madagascar correspond maintenant une vraie île. Ménouthias disparaît alors.

37 Carta d'Alberto Cantino de 1501 (17 oct.) ao duque de Ferrara, Imprimeria Nacional, Lisboa, 1909 ; Charta da navigar per le isole novamente t[rovate] in la parte de l'India dono Alberto Cantino al S. duca Hercole, Il Bulino edizioni d'arte, Modena, 2005 ; P. FrabetTi, Saggio di una illustrazione del planisfero portoghese detto "del Cantino ", en C. Marzoli, Imago et mensura mundi, Atti del IX congresso internazionale di storia della cartografia, vol. 3, Roma, Istituto della Enciclopedia Italiana, 1985, p. 81-88 ; A.T. DA MotA, A Africa no planisferio português anonimo "Cantino ", Junta de investigaçoes do ultramar, Lisboa, 1977. 

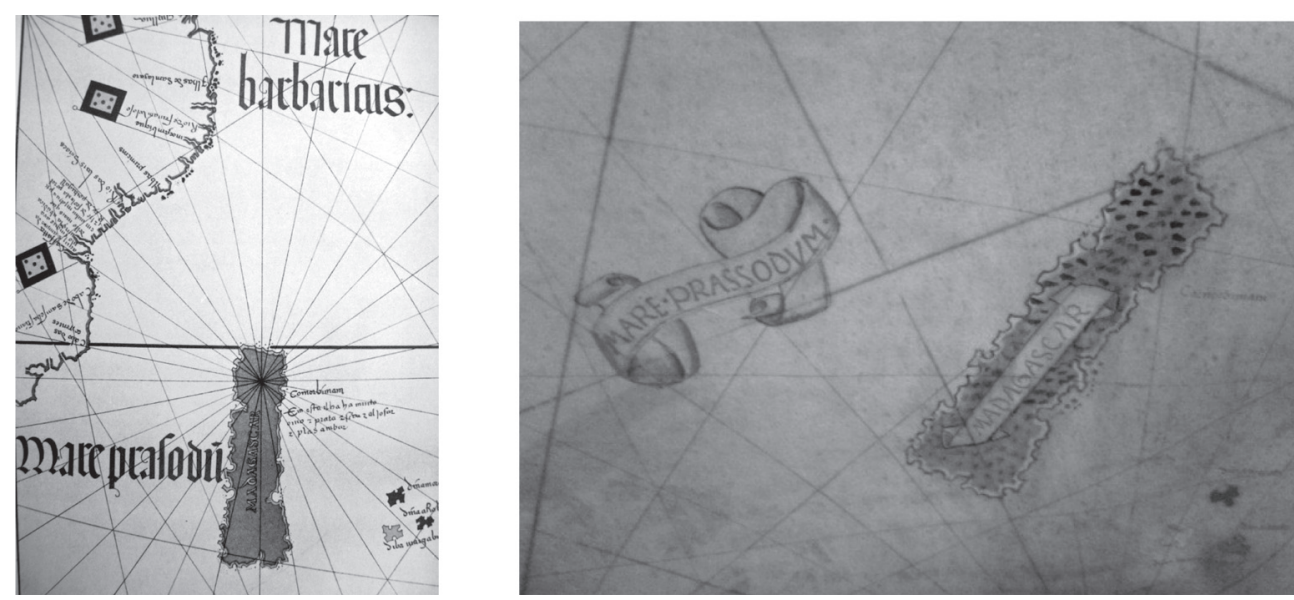

Fig. 6 : Les premières représentations de la vraie morphologie de Madagascar : cartes de Cantino et Caverio (copyright BnF, Cartes et Plans).

La superposition Ménouthias/Madagascar dura toutefois longtemps. Le doute que Ménouthias fût l'île de Sain Laurent avait assailli les navigateurs portugais, comme on peut le lire dans les Decadas da Asia de Joao de Barros/Diego do Couto (Dec VII, livre IV, cap. V, p. 312-314 ${ }^{38}$ ). Ils avaient cependant penché pour une identification de Ménouthias avec une des Comores. Dans la carte de Mercator (1569), au contraire, la filiation Ménouthias de Ptolémée, Madagascar de Marco Polo, Saint Laurent, est explicitée dans une légende :

38 «O nome proprio desta ilha, por que os naturaes a noeão, he Ubuque, e por essa a conhecen os Mouros, que pera ella navegão ; Ptholomeu lhe chama Minuthias, ou presumen os Geografos que he esta a que temos as duvidas que logo diremos. Os modernos lhe chamão Madagascar, e os Portuguezes a ilha de S. Lourenço, porque en tal dia foi descuberta por elles. [...] Tem alguns, como jà dissemos, que esta ilha seja a Minuthias de ptolomeu, ao que nos se offereçeran duvidas ; porque se elle tivera conhecimento desta ilha, tambén não deixára de o dar das Ilhas do Comoró tão nomeadas ; porque se o mercadores daquelle tempo, que lhes podian dar informaçóes por navegarem por toda aquella costa de Melinde, se víram humas, e outras, forçado le houveram de dar relação dellas, e não da de S. Lourenço só. E lançando sobre isso nosso juizo, nos parece que a ilha Angazijá, que he a nord as do Comoró, he a Minuthias ". 


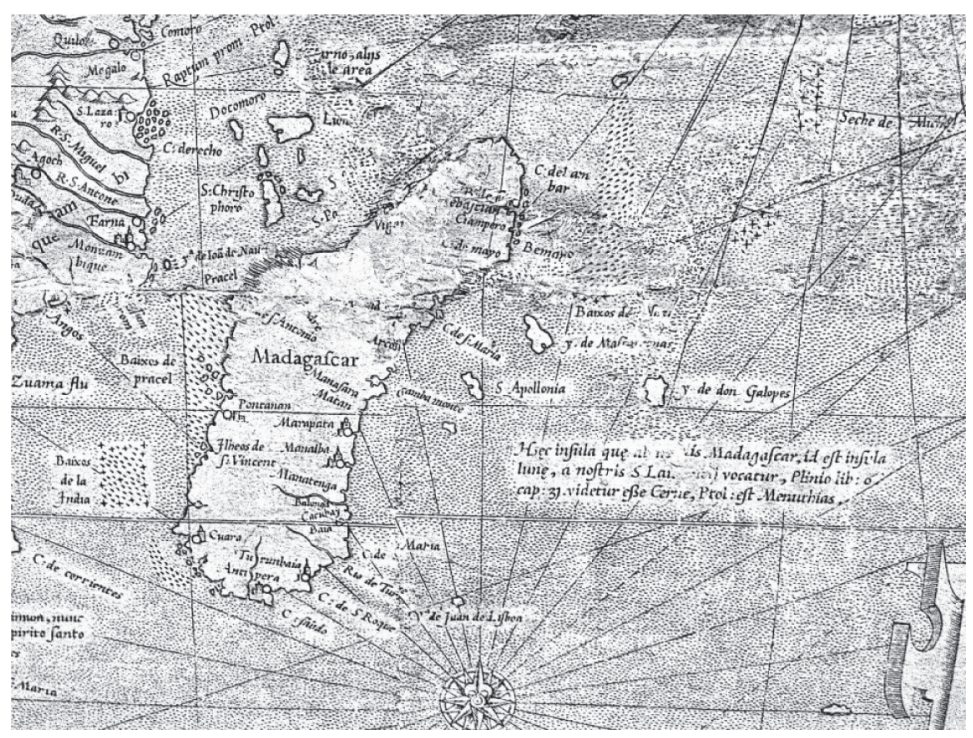

Fig. 7 : Carte de Mercator, Ad usum navigantium, détail, copyright BnF, Cartes et Plans.

Les cartes que nous avons successivement examinées nous portent à la même conclusion que Grandidier : "Il règne, pendant toute la première moitié du XVI siècle, une grande indécision au sujet du nombre et de la situation des îles de l'Afrique Orientale ; les renseignements tirés de la Géographie de Ptolémée et du Livre des Merveilles de Marco Polo, qui ne concordaient pas et qui ne pouvaient pas concorder avec ceux fournis par les navigateurs portugais, puisqu'ils ne s'appliquaient pas aux mêmes pays, ont amené une confusion bizarre et fâcheuse ${ }^{39}$. "

On espère avoir ici éclairé les différentes étapes de cette confusion toponymique « bizarre et fâcheuse ". Madagascar, en effet, reçut assez tard son nom " officiel ». Au bout de ces péripéties cartographiques, l'île « errante ", devint enfin une terre concrète à explorer.

Bianca Maria Altomare

Université de Reims-Champagne-Ardenne

Maison de la Recherche

57, rue Pierre Taittinger

51096 Reims Cedex

bianca.maria-altomare@univ-reims.fr

39 Grandidier, Histoire de la géographie, p. 34. 